\title{
The design of the intelligent alarm ring foot
}

\section{Jiahui Chen}

Department of Electronic and Communication Engineering, North China Electric Power University, Baoding 071000, China

www.cjh161514@qq.com

Keywords: stm32, ADXL345, weight matrix

\begin{abstract}
This paper introduces an intelligent alarm ring foot which is based on the stm32. The device hardware mainly includes data acquisition circuit, data storage circuit, control circuit and data communication circuit. Analyzing data from the ADXL345 three-axis acceleration sensor and calculating by the weight matrix .The device can identify specific alarm action (Stomping three times) and communicate with app. After testing, the device can identify specific action fast and accurate.
\end{abstract}

\section{Introduction}

The traditional intelligent equipment for help has some drawbacks. For instance, the means of distress is too simple and distress signal is easily interrupted. This article proposed an intelligent alarm foot ring indirect trigger signal by stomping three times. The single chip microcomputer deliver signal to the phone. The phone sends real-time location timely and updates their location constantly. So as to achieve the purpose of protecting the wearer's life safety.

\section{The overall scheme design}

This system consists of data acquisition module, data storage module, data communication module and power module. System block diagram is shown in figure 1. First, the ADXL345 is as the data acquisition source of the algorithm in this paper. And sent the signal to STM32 for analysis and processing. HC-05 master and slave integrated Bluetooth module is a bridge between the SCM and phone. When the microcontroller identify a specific rescue action, getting in touch with the phone to send their own real-time location by the Bluetooth. And the trigger data stored in the external flash chip W25Q64, for later calibration.

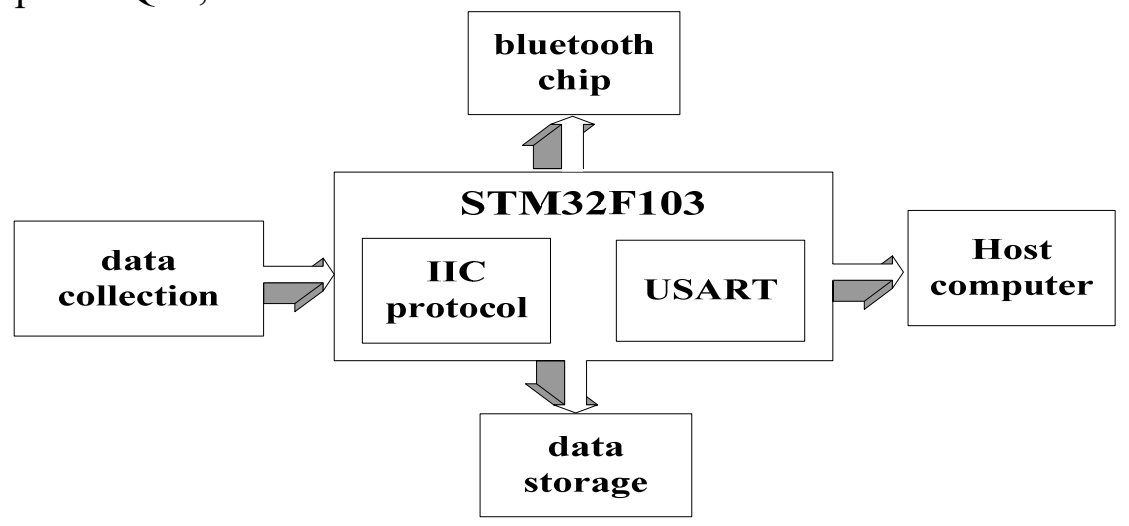

Fig.1 System principle

\section{System hardware design}

\section{Stm32 on-chip resources.}

The system uses ST's 32-bit enhanced microprocessor STM32F103 based on the Cortex-M3 core as the control core. It has the advantages of high performance and low power consumption. The maximum operating frequency of the chip can reach $72 \mathrm{MHz}$, with $512 \mathrm{~K}$ bytes of flash memory 
and $64 \mathrm{~K}$ bytes of SRAM.

\section{Date collection.}

Data acquisition part use the low power consumption, LGA package three-axis acceleration chip ADXL345. In the supply voltage of $3.3 \mathrm{~V}$, in measurement mode the current is as low as $40 \mathrm{uA}$. And in standby mode the current is only $0.1 \mathrm{uA}$. It has a high resolution (13 bit), high measurement range in gravitational acceleration $( \pm 16 \mathrm{~g})$. Its external circuit is shown in Figure 2.

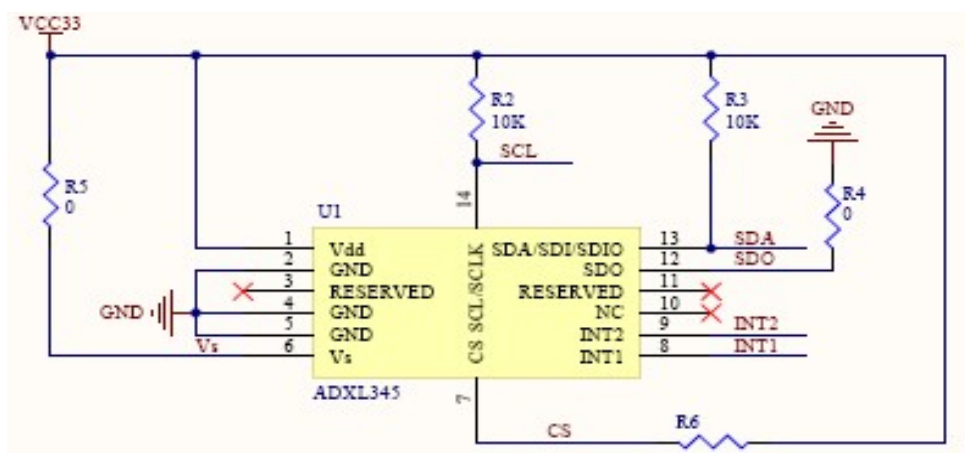

Fig.2 ADXL345 external circuit

\section{Data storage.}

In data storage adopts the low-power flash chip W25Q64. By using the SPI protocol to driver it. The working current is only $4 \mathrm{~mA}$.

\section{Data communication.}

In this text, data communication is adopting the CRS Company's Bluetooth chip. It provides UART interface, SPI interface, PCM interface, and PIO interface. Its operating voltage is 3.3V. Serial port baud rate support multiple choice, the default is $9600 \mathrm{~b} / \mathrm{s}$. The microcontroller can communicate with the mobile app via the Bluetooth chip.

Power supply part.

The power chip choose LDO chip LM1117 for single-chip and sensor power supply.

The consumption of LM1117 is small, with a good load capacity and temperature characteristics, stable output $3.3 \mathrm{~V}$.

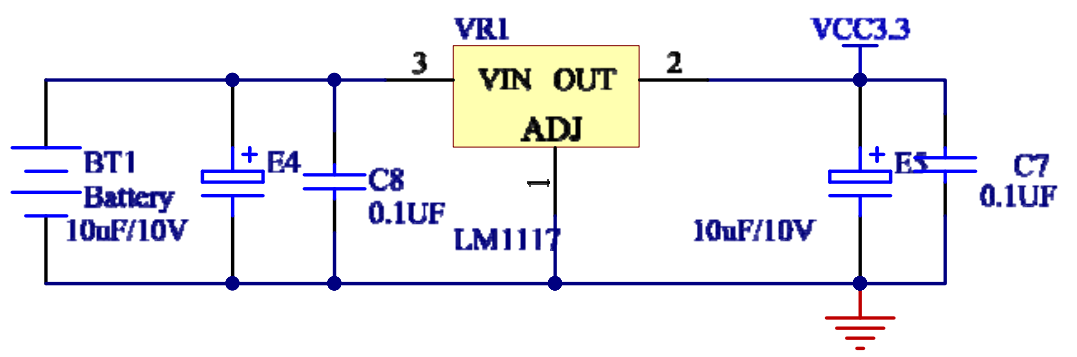

Fig.3 power supply schematic circuit

\section{System software design}

\section{Software design flow.}

After it is power on. IO port, data acquisition module, data storage module and communication module are to be initialized. Then the data acquisition module enters the operating mode. When microcontroller recognizes the specific action, it would trigger interrupt. Bluetooth and mobile app to get in touch, achieving the alarm function. The software design flow chart is shown in Figure 4. 


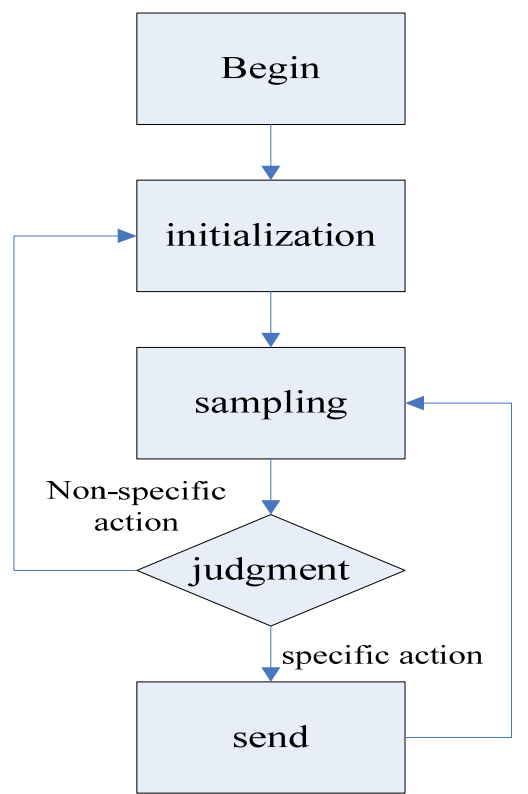

Fig.4 Software design flow chart

\section{Action recognition algorithm.}

The algorithm of action recognition is mainly based on the establishment of BP neural network model. Before the application, it needs for continuous learning, in order to achieve the best results. Through the back of the error propagation, and adjust the weight constantly that is according to the error, and finally the actual output and the desired output error achieve the desired accuracy. The actual operation steps are divided into acceleration data acquisition, acceleration data preprocessing, action data interception, action feature extraction and motion recognition. The feature quantity is as an input, and the action is effectively recognized according to the trained weight matrix. The data is stored in flash and is used to update the weight matrix. The steps are shown in figure 5.

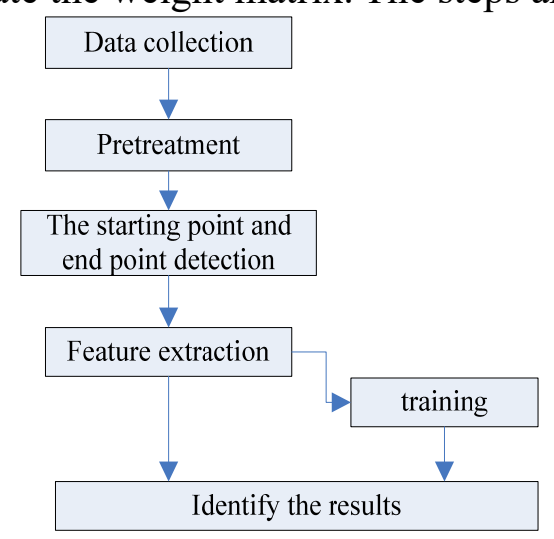

Fig.5 Identify flow diagram

\section{The System Test}

Set the threshold for the recognition action after the data calculated by the weight matrix. The table represents the training results of the different motion states through the weight matrix and the mean filter.

Table.1 Data collected by different actions

\begin{tabular}{|c|c|c|c|}
\hline & the first & the second & the third \\
\hline Walking slowly & 971 & 923 & 811 \\
\hline Brisk walking & 1014 & 1509 & 1716 \\
\hline Running & 1657 & 1833 & 1790 \\
\hline Continuous stomping & 2386 & 2102 & 1993 \\
\hline
\end{tabular}

In the testing process there are some errors due to the different testers. But the overall training results of specific actions (Stomping three times) have a certain degree of identification. 


\section{Conclusion}

In this paper, designing a specific action to identify and alarm the foot ring through the analysis of the acceleration sensor data collected. The foot ring meet the compact characteristics of wearing equipment, and achieving a low-power design. Foot ring identify the specific action accurate and fast, can be better to ensure personal safety.

\section{References}

[1]. Yuan Xiuping (Coll. of Inf., Shanghai Normal Univ., Shanghai, China); Li Jia-Nan; Fang Zuhua Source: 2015 8th International Conference on Intelligent Computation Technology and Automation (ICICTA), p 446-9, 2015

[2]. J.N. Wu,"Novel Design of Remote Real-time Monitor System for Community Medical Healthcare Based on Wireless Sensor Network", Computer Systems \& Applications. China, vol. 23, pp. 77-85, Feb 2014.

[3]. S.R. Hill, Improving Healthcare Service Quality and Patients' Life Quality Through Mobile Technologies: The Case of Diabetes Self-management, Lean Thinking for Healthcare, New York: Springer, 2014, pp. 345-359.

[4]. Ruth E. Mayagoitia Anand V. Nene Peter H. Veltink "Accelerometer and rate gyroscope measurement of kinematics: an inexpensive alternative to optical motion analysis systems" Journal of Biomechanics vol. 35 no. 4 pp. 537-542 2002. 